\title{
5. Franchising Empire
}

\author{
Parker Brothers, Atari, and the Rise of LucasArts
}

Stefan Hall

Video games are everywhere. In watches, cell phones, handheld units, on a variety of home consoles and computer operating systems, and within arcades, played by solitary gamers as much as by groups of friends and strangers, in private and in public, across computer networks and even at professional video-game competitions. While video games have been the subject of, or have played a significant role in, films or have been adapted into films, the examination of films turned into gaming properties is a rich topic because of the ways that films and video games converge and diverge. When the topic of film and video games is mentioned, people tend to think about the adaptation from video games to film, yet considering films that have been the source for video game adaptation yields a particularly interesting category for video games. Films continue to prompt the development of many games; the influence here is as much visual as it is conceptual (in terms of characters and narrative) and economic.

With the introduction of the second generation of home consoles (19761983), companies inside and outside of the video game industry sought to harness improved technology to create properties based on adapted media. From arcade ports to comic-book and film adaptations, the video-game industry sought connections with media sources that inspired transmedia design in the past and also sought to make these more formal as a burgeoning business sector through contracted commercial ventures. Given the necessarily visual nature of video games, an apparent source of rich material for adaptation existed in film. As both the video-game industry and film industry sought to find ways to license existing media properties, fantastic genres readily emerged as a rich source to be tapped, and Star Wars proved to be a particularly lucrative property for adaptation by game designers.

Parker Brothers, a toy and game manufacturer known for publishing major hits such as Sorry! (1934) and Monopoly (1935), entered the home console video game market in 1982. The company had found success in 1978 with the electronic handheld game Merlin, selling the entire production run of 700,000 units before Christmas and reaching \$100 million in sales the 
following year. ${ }^{1}$ But this success was short-lived, as the handheld game market quickly became oversaturated and sales began to fall. Early in 1981, the head of Atari's home computer division approached the senior vice president of research and development at Parker Brothers to ask if Atari could license some Parker Brothers board games for home cartridge versions. Having flirted with success in electronic handheld games, Parker Brothers decided Atari's interest was evidence enough that they should develop their own games. What made the entry of Parker Brothers different from that of other third-party software producers was the fact that they were an established gaming company with a significant research and development division to help ease the transition from physical to digital games.

At a cost of $\$ 50,000$, Parker Brothers reverse-engineered Atari's hardware, enabling them to move into cartridge production independently. ${ }^{2}$ While game development was being pursued at Parker Brothers, parent company General Mills investigated two other possible scenarios for entry into the video-game market. The marketing and design office advocated a new video-game system to compete with Atari, which would require a tremendous commitment of resources. Kenner proposed licensing properties such as Star Wars action figures and Strawberry Shortcake to Atari in order to minimize risk of developing a rival game system, but Parker Brothers presented a compromise to program their own cartridges internally, first for the Atari console, then for the Intellivision, and finally for home computers. ${ }^{3}$ Since General Mills owned both Kenner and Parker Brothers, it was relatively easy for Parker Brothers to obtain the game development rights and essentially outmaneuver Twentieth Century Fox on their own film property. ${ }^{4}$ The excitement at the debut of Frogger and the film-to-game adaptation Star Wars: The Empire Strikes Back (SW:ESB) at the 1982 Toy Fair prompted Parker Brothers to revise its initial profit forecast of $\$ 15$ million several times over and to create at least four other titles for release that year.

1 Philip Orbanes, The Game Makers: The Story of Parker Brothers from Tiddledy Winks to Trivial Pursuit, (Boston: Harvard Business School Press, 2003), 174-75.

2 Sydney Ladensohn Stern and Ted Schoenhaus, Toyland: The High-Stakes Game of the Toy Industry (Chicago: Contemporary Books, 1990), 259.

3 Orbanes, The Game Makers, 181.

4 Twentieth Century Fox became the first film studio to specifically create a video game development studio when it established Fox Video Games as a subsidiary, first by licensing games from Sirius Software and then using titles in their distribution catalog as the basis for video games, including MegaForce (1982), Fantastic Voyage (1982), and Spacemaster X-7 (1983). 
From June through December 1982, cartridge sales ultimately generated $\$ 74$ million in revenue for Parker Brothers. ${ }^{5}$

Given the technological limitations of the time, $S W: E S B$ did not attempt to replicate the entire film's narrative, as later film-to-game adaptations did, but took a particular moment and developed a game around it. In this case, the Empire's memorable assault on the Rebels' stronghold on Hoth was especially useful. Parker Brothers marketing manager Bill Bracy recalled the process of choosing the topic of the adaptation as coming from a small group "including traditional game designers, video game players, and a couple of us in marketing brainstorm[ing] on the various scenes from The Empire Strikes Back" and "developing storyboards and experimenting with game techniques." 6

By limiting themselves to the Hoth battle, the development team at Parker Brothers created a side-scrolling shooter that fit nicely into a familiar genre. In dealing with the memory restrictions of home consoles, the most detail is devoted to the vehicles, which - though blocky-are still easily recognizable. Scale is also important, with the player's snowspeeder dwarfed by the size of the AT-ATs, providing an effect not typically seen in games at the time, as the player and the opponents were often equal in size. The use of parallax scrolling between the mountain range in the background and the surface ice in the foreground creates a distinctive depth of field. Finally, the iconic theme from the John Williams score, synthesized through the platform's audio hardware, is played when the game is first started and again every two minutes for as long as the player survives the attack. Surviving then prompts a 20-second audio clip of the main title theme that functions as a power-up, imbuing the player with the Force and becoming invulnerable to enemy attack. But as viewers of the film surely know, the Rebels are still defeated in the end. ${ }^{7}$ Any sort of success in the game therefore comes not from changing the narrative established by the film, but in delaying its outcome as long as possible.

5 Orbanes, The Game Makers, 186.

6 Nick Montfort and Ian Bogost, Racing the Beam: The Atari Video Computer System (Cambridge: MIT Press, 2009), 128.

7 Noted author Harlan Ellison wrote an infamous review of the $S W$ :ESB game in 1982 for the September issue Video Review magazine in which he likened the repetitive gameplay to the myth of Sisyphus, describing the "inspired exploitation of the Star Wars totem in videogame form [...] as the most virulent electronic botulism," and generally decrying the game because it did not allow for the possibility of winning (since the player will either run out of ships or the power generator will be destroyed). Harlan Ellison, "Rolling Dat Ole Debbil Electronic Stone," Sleepless Nights in the Procrustean Bed (San Bernardino: Borgo Press, 1984), 70. 
While gamers had already become used to losing all of their lives in a game and, indeed, many games of this time have no ultimate, resolvable goal of victory, the ludic pleasure of gameplay clearly trumps the usual sense of (narratological) victory.

$S W: E S B$ is a key text in Star Wars's transmedia history and indeed in the history of video games because it is considered the first official licensing of a film-to-game property for home consoles. ${ }^{8}$ Produced for both the Atari Video Computer System (VCS) and the Mattel Intellivision, the game also functioned as a cross-platform title. This business model differed from the exclusivity that Atari later promoted with its film-to-game properties; the decision to promote a title as exclusive to a particular console or to diversify across several different hardware platforms is central to the contemporary video game industry, particularly as significant and potentially lucrative endorsement deals are imbricated in these decisions. For a company entering the video game market for the first time, the decision for Parker Brothers to provide cross-platform support gave them an advantage over Atari and Mattel, who only produced games for their own systems, while demonstrating that Parker Brothers was making a serious commitment to video games. ${ }^{9}$ Although the game adaptation post-dated The Empire Strikes Back by two years, the popularity and success of SW:ESB also set the precedent for video-game developers to license games based on films that had been released years (or even decades) earlier.

Two subsequent titles-Star Wars: The Arcade Game and Star Wars: Return of the Jedi: Death Star Battle - were produced by Parker Brothers for the Atari 2600, with cross-platform versions for the Atari 5200. An additional 2600 title, Star Wars: Jedi Arena, based tangentially on A New Hope's lightsaber training droid sequence, was released by Parker Brothers, with another Return of the Jedi title (Ewok Adventure) developed but never released. The release of $S W: E S B$ "shows how a compelling cinematic situation can be translated effectively into a videogame challenge. ${ }^{{ }^{10}}$ It also initiated the most prolific franchise in video-game history, in addition to being the most licensed film property, with over 120 unique titles appearing

8 The first game to be made and released in conjunction with a theatrical film was TRON, released in 1982 several months before $S W: E S B$. TRON was an arcade game before being ported to a series of titles for the Atari and Intellivision consoles.

9 At the time that support for both systems was announced, Parker Brothers had only possessed the capability to program for the Atari. The engineers quickly employed their reverse-engineering tricks with the Intellivision and would later go on to do the same with the Colecovision platform and several home computer systems.

10 Montfort and Bogost, Racing the Beam, 16. 
in the 35 years following the release of $S W: E S B$ across all available platforms and ranging across a wide variety of genres.

Having observed the success of $S W: E S B$, other companies quickly began to produce games based on film properties, including Atari. It scored two of the biggest film licenses in those years when Atari acquired the home game rights to the Steven Spielberg blockbusters Raiders of the Lost Ark (1981) and E.T. the Extra-Terrestrial (1982) for a combined \$20 million. ${ }^{11}$ Atari also sought out other agreements in 1982, gaining the rights to arcade games based on the Star Wars license, as the Parker Brothers contracts had only covered home console cartridges. While Parker Brothers had outmaneuvered Atari on the console front, Atari had something Parker Brothers did not: an established coin-op division, which was the foundation of Atari as a company. ${ }^{12}$

Star Wars: The Arcade Game (SW:TAG) was a first-person flight simulator released two months after Return of the Jedi hit theaters, despite being a loose adaptation of a scenario from A New Hope. ${ }^{13}$ SW:TAG featured digitized audio effects lifted from the film, including voice samples of Luke Skywalker, Darth Vader, Han Solo, and Obi-Wan Kenobi, in addition to the main title theme. It had originally been developed as a game called Warp Speed, designed by Ed Rotberg. When Rotberg left Atari, his game was overhauled to produce $S W: T A G$, and most of the original game elements were changed..$^{14}$ The player dogfights with TIE fighters en route to the Death Star, using a yoke controller scheme instead of the typical joystick, and then flies the trench run from the film (see Drew Morton's chapter in this volume). The trench was somewhat different from that of the film and, as the game became more difficult during play, an intermediate level was introduced for the player to fly through involving a run across the surface of the Death Star to reach the trench. High-scoring players were awarded by induction into Princess Leia's Rebel Guard. To further highlight the connection between the game and film, Atari produced a promotional poster for the game in the style of the theatrical one-sheet.

Lucasfilm responded to Atari's initial proposal for the game in an internal memo dated December 15, 1982 by providing a list of seven points to eliminate inconsistencies between the game and the Star Wars storyworld. This

11 Rusel DeMaria and Johnny Wilson, High Score! The Illustrated History of Electronic Games (New York: McGraw-Hill/Osborne, 2004), 99.

12 Additionally, when Parker Brothers ported SW:TAG to home consoles, Atari was able to get a piece of the revenue.

13 John Sellers, Arcade Fever: The Fan's Guide to the Golden Age of Video Games (Philadelphia, PA: Running Press, 2001), 132.

14 DeMaria and Wilson, High Score!, 90. 
includes an explanation of how X-Wings are piloted, celestial mechanics, an in-game rationalization for how deflector shields are charged, and clarification of terms such as "parsecs" and "warp drive" (which is, as the memo points out, a term from Star Trek). In the prototype phase, Atari conducted focus group testing on January 24, 1983: three groups of players-15-19 year-old men, 20-35 year-old men, and 17-30 year-old women-provided interesting feedback about the game. Although its reception was generally positive, the playtesters did raise the question as to why the game was being designed so long after the movie's release date. All groups saw the game as appealing most strongly to male gamers. Another request was for the addition of a sit-down cabinet for added realism and the game was therefore released in both upright cabinet and cockpit (with stereo surround sound) versions. ${ }^{15}$

Lucasfilm was perhaps the most notable movie studio joining the ranks of companies looking to expand their properties through video games in the $198 \mathrm{os}$. With the financial success of Star Wars, fueled by the sale of licensed merchandise, Lucasfilm had the financial power to diversify across other media and so made its first steps into the video-game industry. Its Games Group was founded in May 1982 within Lucasfilm's Computer Division. By cushioning the Games Group within several levels of its corporate structure, Lucasfilm created a sort of contained design environment in what is now commonly referred to as a "game incubator." To further strengthen the Games Group, Lucas signed a joint cooperative agreement with Atari, ${ }^{16}$ after which Atari invested $\$ 1$ million into the Games Group. ${ }^{17}$ According to Robert Doris, Vice President of Lucasfilm and general manager of the Computer Division, the company's movement into the video-game business was a calculated result of the perception that video games were becoming increasingly cinematic. While this was an advantageous deal for both parties, Lucasfilm used Atari as a testing bed for video-game development, taking several years to release the first two games-Rescue on Fractalus! and Ballblazer for the 5200 and the 7800 game systems — because "[a]mid this convergence of technology and entertainment, it was apparent that the core target audiences of special-effects movies and the Atari game

15 Interestingly, according to an inter-office memo dated August 2, 1983, Atari may have considered a Star Wars II game that would essentially have linked games, in a sort of primitive multiplayer system, to give players a squadron attack experience.

16 Michael Ciraolo, "Lucasfilm \& Atari: Creative Partners," Antic 3, no. 4 (1984): 40.

17

DeMaria and Wilson, High Score!, 198. 
platform had significant similarities. ${ }^{318}$ The Games Group began by creating basic $3 \mathrm{D}$ point-of-view $(\mathrm{POV})$ routines as an experiment, and then turned to studying the dedicated chips and microprocessor of Atari's computer. ${ }^{19}$ Since $3 \mathrm{D}$ computer modeling was barely in its infancy, the Games Group had Industrial Light \& Magic create scale models of the ships in Ballblazer and Rescue on Fractalus! using the same process as the Star Wars films. This gave the group a thorough understanding not only of the basic game architecture, but also of where the technology could be pushed or "how the form might evolve-ultimately, maybe, to meld with movies themselves. ${ }^{{ }^{20}}$

In 1983, two early versions of the first games-known at that point as BallBlaster and Rebel Rescue - were shown to Atari. ${ }^{21}$ Final versions of the games were delivered in May 1984 and announced at a press conference that began with the opening sequence from A New Hope. ${ }^{22}$ This long period of development enabled Lucasfilm to experience video-game industry operations while only committing a small amount of resources (the Games Group initially only counted six men). The Games Group borrowed heavily from development procedures in the film-production division, including drawing models, making costumes, and writing extensive storylines to develop a narrative underpinning for the games more fully. As Langston explained, "My charter was to figure out where in the entertainment industry to apply the kind of high-tech approaches that were proving so revolutionary in graphics, sound, and editing for movies [...] It took very little time to settle on pursuing games. ${ }^{{ }^{23}} \mathrm{An}$ internal memo written by game designer David Fox in 1982 listed some thoughts on game development, in particular what films do well—such as special effects modeling new realities and creating narrative engagement — and where video games lag behind, noting that

18 Rob Smith, Rogue Leaders: The Story of LucasArts (San Francisco, CA: Chronicle Books, 2008), 11 .

19 Peter Langston, “Ten Tips from the Programming Pros: Secrets from Lucasfilm's Game Group," Atari Connection (Spring 1984): 34.

20 Associated Press, "Lucas Looks beyond Film-Will Interactive Movies Try to Eat Your Popcorn?” Access (Fall 1984), 12.

21 During testing of Rebel Rescue, Lucas looked for a fire button on the controls. In the game's fiction, it was explained that the weapons had been removed to make more room for the marooned pilots to be rescued. Lucas asked Fox if that was a part of the game design or a moral choice. This is an important question to raise, and the introduction of moral choices as part of the play mechanics usually took the form of alternate endings based on what actions the player had or had not done. Offering different narrative outcomes was initially far easier to do on computers and helped to distinguish them from other gaming platforms.

22 Jonathan Greer, "Star Wars' Creator Teams Up with Atari," San Jose Mercury News, May 8 $1984, \mathrm{Gl}$.

23 Smith, Rogue Leaders, 12-13. 
they were not taking full advantage of the hardware, including video and audio); that they sometimes seemed constrained in game mechanics due to genre; and that they did not offer a high level of escapism. ${ }^{24}$

For many years, Lucasfilm Games would eschew developing its own Star Wars games, preferring to license out the franchise instead. By Lucas's own admission, "I wanted to have a really creative, independent shop. It's not a coincidence that our early video games were not based on the Star Wars or Indiana Jones films. ${ }^{25}$ In 1990, a reorganization of the Lucas companies made Lucasfilm's Games Group a part of the newly created LucasArts Entertainment Company, which included Industrial Light \& Magic and Skywalker Sound. After achieving a level of success with Indiana Jones games, Lucasfilm Games finally moved on to creating its own Star Wars game after licensing the property to other developers for several years. Because the publishing rights were still owned by JVC, the first Lucasoriginated Star Wars games had some insulation against risk should something go awry. To add extra insurance, Lucasfilm Games handled design and production, while subcontracting the actual programming to Beam Software, an outside studio based in Australia. ${ }^{26}$ The deal with JVC included six games to be developed for the Nintendo Entertainment System (NES), with JVC advancing $\$ 1$ million and each game budgeted at $\$ 80,000-\$ 100,000$ for development, leaving a financial cushion to handle any development issues. As production on Star Wars (the game named simply after the first film's original title) commenced with an eye on a September 1990 release date, an issue with the limited memory capacity of the NES arose. Therefore, the drive "to re-create every significant Star Wars-movie action scene was abandoned in favor of fitting key gameplay scenes into the $128 \mathrm{~K}$ available on the NES. ${ }^{\prime 27}$ Like the Parker Brothers version of SW:ESB, the game designers had to decide what constituted an essential experience of the film and put that into some sort of playable system.

Side-scrolling platformers were popular at the time, so the game was designed for the player to primarily control Luke Skywalker — with Princess Leia and Han Solo used for certain missions - and concentrated on distinct locales such as Tatooine and the Death Star. To diversify the play experience

24 Fox also raised a question regarding the licensing agreement of Star Wars, as the title of Rebel Rescue had to be changed because of its loose connection to the Star Wars universe, wondering if games might be set in the world of the films that reference places, vehicles, and weapons, but provide new characters and stories.

25 Smith, Rogue Leaders, 7.

26 Smith, Rogue Leaders, 72.

27 Smith, Rogue Leaders, 73. 
further, a first-person asteroid belt mission and a top-down Death Star trench run sequence were added. Between the popularity of the NES and the recognizability of the Star Wars brand, the game sold well and production on The Empire Strikes Back began soon thereafter in collaboration with Utah-based Sculptured Software as subcontracted developer. This game essentially followed the same formula as its predecessor and attempted to identify core cinematic referents (locations like Hoth, Dagobah, and Bespin, and characters like Darth Vader, Boba Fett, and Yoda) while introducing some gameplay not seen in the film, such as fighting the cloud cars of Cloud City and racing against Boba Fett's Slave I.

The decision to include these extra-cinematic play elements opened the door to questions of authenticity and canon for this incarnation of the franchise. This is an especially notable issue for Star Wars in that the films are often seen as the source from which all other stories derive. ${ }^{28}$ While Lucas had final say over all Star Wars products, and the EU tried to stay consistent at the time with the elements of the films, licensed media properties generally had to remain aligned with the overall canon while also offering experiences to their audiences that utilized the best aspects of each medium. Predominantly for video games, this meant striking a balance between the narrative, the look of the game, and available gameplay options. ${ }^{29}$ This issue becomes especially important for film-to-game adaptations that attempt to enlarge the world presented within the film.

While TESB was in development in 1991, Nintendo released the Super NES (SNES), whose increased hardware potential piqued the attention of Lucasfilm Games. A decision was therefore made to develop most of the game in-house, with Sculptured Software providing technical implementation. Both Super Star Wars (1992) and Super Star Wars: The Empire Strikes Back (SSW:ESB, 1993) were praised for taking advantage of the graphics capability at the time, as well as being fun to play. ${ }^{30}$ Like The Empire Strikes Back on the NES, SSW:ESB also veered away from what was canonical as far as the film showed, with part of the rationale being that the game's

28 The EU was especially important in the 1990s. In particular, Timothy Zahn's Thrawn Trilogy novels (1991-1993) renewed interest in the Star Wars brand, further enabling the video games to capitalize on the franchise's cultural appeal in the absence of any theatrical films.

29 For example, the fighting game Masters of Teräs Käsi (1997) goes to great narrative lengths to explain why Star Wars characters are pitted in arena-style combat and, for this reason (as well as bad game controls), it was widely regarded as a flop.

30 One of the advantages of the SNES was Mode 7 , a texture-mapping technique to change rotation and scale and thus provide a sense of $3 \mathrm{D}$ perspective, which allowed game designers to more closely emulate the look of a movie world. 
"visual effects created a clear separation in the audience's mind between a small-screen interactive game and a big-screen blockbuster. ${ }^{131}$ While this sounds somewhat plausible given the severe technological limitations in terms of video games' degree of cinematic realism in the early 1990s, this particular distinction between a game and a film has since been disrupted with the most current capabilities of gaming technology.

The success of the NES and SNES Star Wars titles gave Lucasfilm Games the clout to restructure its distribution deals and eliminate the need for external publishers. For the newly rebranded LucasArts, technological issues again pushed game design, specifically in CD-ROMs. While the standards for using compact discs as a computer storage medium had been established by Sony and Philips in 1985, practical and affordable distribution to the home user took several more years. Rebel Assault's release in 1993 was a primary reason why many home computer users decided to upgrade to computers equipped with a CD-ROM drive. ${ }^{32}$ The CD-ROM's storage potential not only meant that game developers could create more sophisticated programs that would offer improved graphics and longer play experiences, but they could also incorporate live-action footage seen in full-motion video (FMV) games such as The 7 th Guest (1992) and Voyeur (1993). The designers of Rebel Assault decided to film new live-action footage set in the Star Wars universe and this development caught Lucas's attention:

If video was suddenly the primary visual production method, the games would start to encroach on the movie space. According to producer Hal Barwood, Lucas was concerned that if movie-like footage was shot, it could cause confusion with the film canon, and players would assume Lucas was somehow at the helm. ${ }^{33}$

The live-action footage was instead combined with overlaid sprites that created sophisticated visuals that still remained distinct from cinematic photorealism. ${ }^{34}$ Memory constraints and processing power also restricted the free movement and sense of exploration that players experienced in other games by necessitating that the action largely remain confined to a

31 Smith, Rogue Leaders, 74.

32 Mark Langshaw, “'Star Wars: Rebel Assault' Retrospective: A Force to Be Reckoned With," Digital Spy, April 6, 2013, accessed May 15, 2017, http://www.digitalspy.com/gaming/retro-gaming/ news/a470859/star-wars-rebel-assault-retrospective-a-force-to-be-reckoned-with/.

33 Smith, Rogue Leaders, 78.

34 Live-action scenes would be used again in Rebel Assault II (1995) and even more so-an entire hour's worth—in Jedi Knight: Dark Forces II (1997). 
limited combination of interactions, making the game function like a rail shooter. Finally, the game design provided a play experience that lasted only a few hours, roughly equivalent to a Star Wars movie, thus also keeping development costs more manageable.

Despite these limitations, Rebel Assault's look and style helped make the game a major success. Before its release, CD-ROM games sold an average of 10,000 copies, but the first-day shipment of Rebel Assault moved 110,000 units; the game ultimately reached sales of over a million copies. ${ }^{35}$ While its popularity certainly could be attributed partly to the Star Wars brand itself, the gameplay also suggested a more cinematic experience and indicated another direction for game design as the hardware advanced. As Lucas noted in a March 3, 1994 memo to Rebel Assault's development staff:

By taking "Star Wars" into the CD-ROM platform you've extended my original story ideas into a new and different form of entertainment. You seem to have set the standard for what this new medium can accomplish for interactive entertainment. It's great that we can also set the standard for what sales can be as well. ${ }^{36}$

The game's influence, particularly in its use of CD-ROM technology, helped the LucasArts developers free themselves from the memory restrictions of computer-system storage at the time and gave them new ideas for game development.

In the late 1980 s and early 199os, Lucasfilm Games developed a series of military simulation games, beginning with Battlehawks 1942 (1988), a WWII trilogy created by a team led by Lawrence Holland. Holland drew upon this experience for his next LucasArts project, the $X$-Wing series (1993-1999). ${ }^{37}$ This EU series attempted to simulate the film experience of starfighter combat faithfully, which itself was based on gun-camera dogfight footage Lucas had studied while making A New Hope.

In the early titles- $X$-Wing, released in 1993, with the expansions Imperial Pursuit and $B$-Wing following the same year-the player worked for the Rebel Alliance. These games begin before the events in A New Hope and involve missions in which allies must be located and the Death Star plans

35 Smith, Rogue Leaders, 78.

36 Smith, Rogue Leaders, 80.

37 Beginning in 1995, Michael Stackpole, collaborating with Mike Baron, produced a popular $X$-Wing: Rogue Squadron comic book for Dark Horse. A year later, Stackpole, along with Aaron Allston, also produced the popular $X$-Wing novel series for Bantam Spectra. 
must be delivered to Princess Leia. The action then moves to intersect with the film and the attack on the Death Star. Players then deal with the evacuation of Yavin IV, eventually arriving on Hoth shortly before the events in The Empire Strikes Back. The game was another commercial success that gave players a new opportunity to fly spaceships within the Star Wars universe.

Additional Star Wars games opened up the possibilities for fan-player engagement with the storyworld by allowing players to fly for the Galactic Empire in TIE Fighter, a move that highlighted the conception of morality in the Star Wars universe, which began as something fairly nuanced before becoming more dogmatic in later Star Wars titles..$^{38}$ According to Holland, "In most great works, the villain or evil side has a lot of intrigue, and we wanted to design with that in mind. We gave the Empire shades of gray, and gave the player a chance to achieve some nobility. ${ }^{39}$ The game's narrative begins after the Rebels' routing on Hoth, continuing through a series of missions in which the player must not only fight the Rebels but help to quell civil wars, dispatch pirates and traitorous Imperials, complete secret objectives for the Emperor, and generally try to keep the peace, albeit through heavily militaristic means.

The first and last games in the series-X-Wing (1993) and X-Wing Alliance (1999) — paralleled events in the original Star Wars trilogy, including endgame missions that recreate the attacks on the first and second Death Stars, despite the fact that Holland, with $X$-Wing, "didn't want to do the movie over again, though, but put in the familiar elements in a similar story."40 Most of the games feature voice acting, which was somewhat unusual for the time, and animated cut scenes (supplemented with occasional computer rendering) that advance the storyline, as well as feature music from the original trilogy. Like $X$-Wing before it, TIE Fighter was a major commercial success, eventually outselling $X$-Wing, and benefiting from improvements in the game's programming. ${ }^{41}$ These critically and financially successful games released by Lucasfilm Games/LucasArts demonstrated that it was possible for a production company to flourish in the video-game industry, draw upon and repurpose their own internal assets, and adapt films to

$3^{8}$ The ability to fly for either the Rebel Alliance or the Galactic Empire was proposed for $X$-Wing but dropped due to programming and time constraints.

39 DeMaria and Wilson, High Score!, 203.

40 DeMaria and Wilson, High Score!, 203.

41 Anticipation for the game was so high that Dodge used it as part of their promotional campaign for the Neon. People who test-drove the car received a demo of the game consisting of one playable level in an unfinished build and an advertisement for the Neon. 
games as appropriate to the medium, often while pushing the boundaries of existing gaming technology.

Even as LucasArts continued to develop new film-to-game Star Wars titles, the older titles in its catalog continued to be profitable. In this regard, despite some occasional technical upgrades, the gameplay remained engaging even if the graphics had grown dated by contemporary standards. Although its film-to-game properties were confined almost exclusively to Star Wars, LucasArts was a useful model for other film studios to consider ways to successfully manage franchise development in video games, not only for adaptations but also for acclaimed original works such as The Secret of Monkey Island (1990) and Grim Fandango (1998). With the 2012 acquisition by Disney and the subsequent dissolution of almost all of the LucasArts staff, the company now exists solely as a licensor of branded IP. It is important, however, to remember the technological and industrial innovations that made LucasArts such a successful and influential company. Considering how quickly change transpires in the video-game industry, it is useful to reflect on this nascent part of its history and consider how earlier developers like Parker Brothers, Atari, and Lucasfilm laid the foundation for much of the medium's future. 
